\title{
Theory of Diffusive Fluctuations
}

\author{
Xinyi Chen-Lin, Luca V. Delacrétaz, and Sean A. Hartnoll \\ Department of Physics, Stanford University, Stanford, California 94305-4060, USA
}

(Received 19 December 2018; published 6 March 2019)

\begin{abstract}
The recently developed effective field theory of fluctuations around thermal equilibrium is used to compute late-time correlation functions of conserved densities. Specializing to systems with a single conservation law, we find that the diffusive pole is shifted in the presence of nonlinear hydrodynamic self-interactions, and that the density-density Green's function acquires a branch point halfway to the diffusive pole, at frequency $\omega=-(i / 2) D k^{2}$. We discuss the relevance of diffusive fluctuations for strongly correlated transport in condensed matter and cold atomic systems.
\end{abstract}

DOI: 10.1103/PhysRevLett.122.091602

Introduction.-Diffusion was invented by Fourier to describe the dynamics of heat [1]. Heat or energy transport is ubiquitous and of relevance to essentially any physical system at nonzero temperature. In modern parlance, diffusion is understood more generally as the universal latetime hydrodynamic description of systems governed by a single conservation law, barring spontaneously broken symmetries. The diffusion constant may itself depend on the background value of the quantity that is being transported. Classically, this results in the diffusion equation being augmented to a nonlinear differential equation and can lead to rich phenomena (see, e.g., [2]), in analogy with turbulence in the Navier-Stokes equation. At the quantum or statistical physics level, the consequence of these nonlinearities is that the effective theory of hydrodynamic fluctuations is interacting.

The traditional approach to address this class of problems is to couple the degrees of freedom (d.o.f.) of interest to stochastic noise fields and solve perturbatively a nonlinear Langevin equation [3,4]. This approach is also familiar in the context of the Kardar-Parisi-Zhang equation [5]. These methods have revealed striking effects in hydrodynamics such as longtime tails [4,6-8] and potentially large renormalizations of transport parameters [9,10]. However, where standard classical hydrodynamic stood on firm symmetry principles [11], the physical principles governing stochastic hydrodynamics - in particular how "noise" fields interact with conserved densities-were less transparent. This was remedied recently by a decade-long effort culminating in a first principles construction of the general effective field theory of hydrodynamic fluctuations

Published by the American Physical Society under the terms of the Creative Commons Attribution 4.0 International license. Further distribution of this work must maintain attribution to the author(s) and the published article's title, journal citation, and DOI. Funded by SCOAP ${ }^{3}$. about a thermal equilibrium state [12-16]; see [17] for a recent review.

Another motivation for a systematic study of hydrodynamic fluctuations is thermalization. The local thermalization (or equilibration) time $\tau_{\text {th }}$ is loosely defined as the time it takes a system to reach local thermodynamic equilibrium. At times $t>\tau_{\text {th }}$, hydrodynamics governs the slower relaxation to global thermodynamic equilibrium. It is tempting to identify the thermalization time with the exponential decay of nonhydrodynamic correlators $\langle\mathcal{O}(t) \mathcal{O}\rangle \sim e^{-t / \tau_{\text {th }}}$. Such correlators are, however, sensitive in general to hydrodynamic longtime tails and therefore strictly do not decay exponentially [4,7]. A better understanding of longtime tails may therefore help provide a sharp definition of $\tau_{\text {th }}$. See, e.g., $[18,19]$ for recent alternative approaches to $\tau_{\text {th }}$.

In the following, we use the general formalism of Ref. [15] to uncover the universal structure of late-time response functions for interacting systems with a single continuous symmetry, focusing on time translation invariance (and therefore heat transport) for concreteness. We find that the thermal dc conductivity and diffusion constant both receive independent nonvanishing radiative corrections, even in the case of a single conserved density, and that the correction is not sign definite. Both of these statements are different to the results obtained from a traditional approach [10], for reasons we shall explain. Moreover, we compute the one-loop retarded Green's function $G_{\varepsilon \varepsilon}^{R}(\omega, k)$ at finite frequency and wave vector, revealing its analytic structure. We conclude by discussing experimental signatures of hydrodynamic fluctuations with applications to insulators, bad metals, and cold atoms.

Formalism.-Our objective is to understand the structure of energy density correlation functions in nonintegrable quantum systems at nonzero temperature

$$
\left\langle\varepsilon(t, x) \varepsilon\left(t^{\prime}, x^{\prime}\right) \cdots\right\rangle_{\beta} \equiv \operatorname{Tr}\left[\rho_{\beta} \varepsilon(t, x) \varepsilon\left(t^{\prime}, x^{\prime}\right) \cdots\right]
$$


where the thermal density matrix $\rho_{\beta}=e^{-\beta H} / \operatorname{Tr} e^{-\beta H}$. Here, we will be interested in the case where energy is the only conserved quantity. The systematic study of a single diffusive charge was initiated in Ref. [15]. In that formalism, furthermore, the contribution of ghosts (or lack thereof) has been well understood [20]. A self-contained review of the formalism with a single conserved charge is given in the Supplemental Material [21]. The output of this method is an effective field theory that provides a perturbative expansion for computing the correlators (1):

$$
Z=\int D \varepsilon D \varphi_{a} e^{i \int \mathcal{L}\left[\varepsilon, \varphi_{a}\right]}
$$

Here, $\varepsilon$ is the energy density and $\varphi_{a}$ is an auxiliary field (the $a$ subscript is not an index). The most general Lagrangian to cubic order in fields was constructed in Ref. [15]. In the Supplemental Material [21] we extend their construction to quartic interactions, which will play a role below. The resulting Lagrangian to leading order in derivatives that is at most quartic in fields is given by

$$
\begin{aligned}
\mathcal{L}= & i T^{2} \kappa\left(\nabla \varphi_{a}\right)^{2}-\varphi_{a}\left(\dot{\varepsilon}-D \nabla^{2} \varepsilon\right) \\
& +\nabla^{2} \varphi_{a}\left(\frac{\lambda}{2} \varepsilon^{2}+\frac{\lambda^{\prime}}{3} \varepsilon^{3}\right)+i c T^{2}\left(\nabla \varphi_{a}\right)^{2}\left(\tilde{\lambda} \varepsilon+\tilde{\lambda}^{\prime} \varepsilon^{2}\right) \\
& +\cdots,
\end{aligned}
$$

where $T$ is the temperature, $D$ the diffusivity, $c$ the specific heat, and $\kappa=c D$ the thermal conductivity. These are all "bare" values that will be renormalized by the interactions in (3). The couplings $\lambda, \lambda^{\prime}, \tilde{\lambda}, \tilde{\lambda}^{\prime}$ themselves can be written as linear combinations of the following derivatives of the transport parameters:

$$
T \partial_{T} \kappa, \quad T^{2} \partial_{T}^{2} \kappa, \quad T \partial_{T} D, \quad T^{2} \partial_{T}^{2} D .
$$

Their explicit expressions are given in the Supplemental Material [21].

The traditional stochastic approach to hydrodynamic fluctuations with Gaussian noise [3-5,7] can be recovered from the general effective action (3) when the interactions that are quadratic in auxiliary fields (i.e., the $\tilde{\lambda}$ and $\tilde{\lambda}^{\prime}$ terms) are absent, by performing a Legendre transform and introducing the noise field $\xi=\partial \mathcal{L} / \partial \varphi_{a}$ [15]. However, when $\tilde{\lambda}$ or $\tilde{\lambda}^{\prime}$ is nonvanishing, the resulting theory will contain interactions of the form $\xi^{2} \varepsilon$ or $\xi^{2} \varepsilon^{2}$. The noise correlations will therefore not be strictly Gaussian because they now depend on energy fluctuations [28].

In the remainder we will show precisely how the interactions in (3) lead to nonanalyticities in response functions and renormalize the transport parameters themselves $[6,8,9,29]$. Concretely, we are interested in the oneloop correction to the retarded Green's function, which is simply diffusive in the absence of interactions

$$
G_{\varepsilon \varepsilon}^{R, 0}(\omega, k)=\frac{i \kappa T k^{2}}{\omega+i D k^{2}} .
$$

The diagrams contributing at one loop are shown in Fig. 1, and computed in the Supplemental Material [21]. These loops are all UV divergent and should be truncated at the hydrodynamic cutoff $k_{\max }=2 \pi / \ell_{\mathrm{th}}$, which defines the thermalization length $\ell_{\mathrm{th}}$. Perturbation theory is controlled because all couplings in the Lagrangian (3) are power counting irrelevant, and therefore have small effects at low, hydrodynamic energy scales. Indeed, the appropriate dimensional analysis is set by the diffusive pole in (5), so that $[\omega]=2[k]$ and $[D]=0$. It follows that $\left[\phi_{a}\right]=[\varepsilon]=$ $(d / 2)[k]$ and hence the cubic couplings $[\lambda]=[\tilde{\lambda}]=$ $-(d / 2)[k]$ are irrelevant. These dimensions suggest that the one-loop corrections to tree-level diffusion, which are quadratic in coupling, will be of the schematic form $D k^{2}\left(1+\lambda^{2} k^{d}\right)$, as we verify below.

Results.-The diagrams shown in Fig. 1 sum up to give the one-loop retarded Green's function

$$
G_{\varepsilon \varepsilon}^{R}(\omega, k)=\frac{i[\kappa+\delta \kappa(\omega, k)] T k^{2}}{\omega+i D k^{2}+\Sigma(\omega, k)}
$$

where both $\delta \kappa(\omega, k)$ and $\Sigma(\omega, k)$ receive analytic and nonanalytic contributions. Separating these contributions as

$$
\begin{aligned}
\delta \kappa(\omega, k) & =\delta \kappa+\kappa_{\star}(\omega, k), \\
\Sigma(\omega, k) & =i \delta D k^{2}+\Sigma_{\star}(\omega, k),
\end{aligned}
$$

one finds that the analytic pieces have the form

$$
\frac{\delta \kappa}{\kappa}=\frac{f_{d}}{c \ell_{\mathrm{th}}^{d}} \lambda_{\kappa}, \quad \frac{\delta D}{D}=\frac{f_{d}}{c \ell_{\mathrm{th}}^{d}} \lambda_{D},
$$

with $f_{d}=\operatorname{Vol}\left(B_{d}\right)=2, \pi, 4 \pi / 3$ for spatial dimensions $d=1,2,3$, and where $\lambda_{\kappa}, \lambda_{D}$ are dimensionless effective couplings. Their explicit form will be given below. The nonanalytic parts of (7) have the form
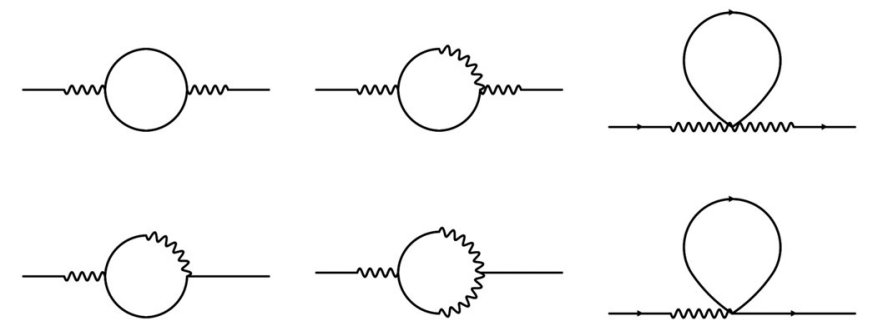

FIG. 1. The one-loop diagrams contributing to $G_{\varepsilon \varepsilon}$. Solid lines denote the energy density field $\varepsilon$ and squiggly lines denote the auxiliary field $\varphi_{a}$. 


$$
\begin{aligned}
\kappa_{\star}(\omega, k) & =f_{\kappa}(\omega, k) \alpha_{d}(\omega, k), \\
\Sigma_{\star}(\omega, k) & =k^{2} f_{\Sigma}(\omega, k) \alpha_{d}(\omega, k),
\end{aligned}
$$

where $f_{\kappa}, f_{\Sigma}$ are analytic functions, shown below, that do not depend on dimension, and the nonanalyticity is

$$
\begin{aligned}
& \alpha_{1}(\omega, k)=\frac{1}{4}\left(k^{2}-\frac{2 i \omega}{D}\right)^{-1 / 2}, \quad(d=1) \\
& \alpha_{2}(\omega, k)=-\frac{1}{16 \pi} \log \left(k^{2}-\frac{2 i \omega}{D}\right), \quad(d=2) \\
& \alpha_{3}(\omega, k)=-\frac{1}{32 \pi}\left(k^{2}-\frac{2 i \omega}{D}\right)^{1 / 2} . \quad(d=3)
\end{aligned}
$$

The effect of these nonanalyticities is suppressed by powers of momenta and frequency appearing in $f_{\kappa}, f_{\Sigma}$, as we will see below.

The retarded Green's function is analytic in the upperhalf frequency plane, as required by causality. The interactions have induced a branch point at $\omega=-(i / 2) D k^{2}$. Moreover, the diffusive pole is split into two poles with small real parts $\omega=-i(D+\delta D) k^{2} \pm O\left(k^{2}|k|^{d}\right)$ [30]. The location of the branch point can be understood from simple kinematics, by putting both internal legs on-shell (in either the retarded or advanced Green's functions) as in Fig. 2. The frequencies $\omega$ for which the on-shell condition is satisfied form a half-line in the complex plane parametrized by the loop momentum $k^{\prime}$, where the Green's function has a branch cut. The branch point is located at the smallest frequency $\omega$ (in magnitude) that can satisfy the on-shell conditions:

$$
\omega_{\star}=-i D \min _{k^{\prime}}\left[k^{2}+2 k \cdot k^{\prime}+2 k^{\prime 2}\right]=-\frac{i}{2} D k^{2} .
$$

In previous treatments, similar physics to what we have just described was found in the coupled diffusion of two modes $[8,10]$. Because of the absence of a systematic
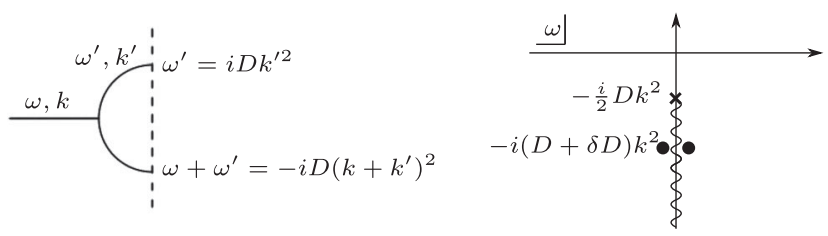

FIG. 2. On-shell condition for the two internal legs (left), and analytic structure of the retarded Green's function $G_{\varepsilon \varepsilon}^{R}(\omega, k)$ at one loop (right). In imposing the on-shell condition, it is important to consider two poles in opposite halves of the complex $\omega^{\prime}$ plane, otherwise the loop contribution vanishes. The pole in the upper half plane arises from an advanced Green's function in the loop: $G^{A}=\left(G^{R}\right)^{*}$. formalism for hydrodynamic fluctuations at that time, those works did not account-among other things-for interactions that are quadratic in the auxiliary field (in particular $\tilde{\lambda}$ ), nor the quartic terms $\lambda^{\prime}$ and $\tilde{\lambda}^{\prime}$. While the systematic approach modifies the results for two coupled modes, see Supplemental Material [21], the most qualitative difference is seen for diffusion of a single conserved density. We have found that renormalization of the diffusion constant and conductivity occurs even in this case, controlled by the effective couplings in (8)

$\lambda_{\kappa}=\frac{c^{2} T^{2}}{D} \tilde{\lambda}^{\prime}, \quad \lambda_{D}=-\frac{c^{2} T^{2}}{2 D^{2}}\left[\lambda(\lambda+\tilde{\lambda})+2 \lambda^{\prime} D\right]$,

which are not sign-definite in general.

Furthermore, we have also found nonanalytic corrections to the Green's function even with a single diffusing mode. These are not as strong as those arising with two modes, as we now explain. The functions $f_{\kappa}, f_{\Sigma}$ appearing in the nonanalytic contributions (9) are

$$
\begin{aligned}
& f_{\kappa}(\omega, k)=\frac{c T^{2}}{D^{2}} k^{2} \lambda \tilde{\lambda}, \\
& f_{\Sigma}(\omega, k)=\frac{c T^{2}}{D^{2}}\left[\omega \lambda(\lambda+\tilde{\lambda})+i D k^{2} \lambda \tilde{\lambda}\right] .
\end{aligned}
$$

While $f_{\Sigma}$ has both the $O(\omega)$ and $O\left(k^{2}\right)$ terms expected at this order in the derivative expansion, $f_{\kappa}$ only has an $O\left(k^{2}\right)$ term. The subsequent suppression of $f_{k}$ as $k \rightarrow 0$ implies that the optical conductivity does not receive nonanalytic corrections, and is instead constant in the hydrodynamic regime

$$
T \kappa(\omega) \equiv \lim _{k \rightarrow 0} \frac{\omega}{k^{2}} \operatorname{Im} G_{\varepsilon \varepsilon}^{R}(\omega, k)=T(\kappa+\delta \kappa) .
$$

This result can be contrasted with the case of two interacting diffusive densities, wherein the optical conductivity receives a nonanalytic fluctuation correction [6,8]. We revisit this case in the Supplemental Material [21], where the analytic structure is discussed in the light of a systematic inclusion of fluctuation effects. We find a nonanalytic correction to the optical conductivity of the form (we use $\sigma$ to denote a generic conductivity)

$$
\delta \sigma(\omega, k) \sim \omega \alpha_{d}(\omega, k)+\cdots,
$$

where $\alpha_{d}$ is as in (10) and $\cdots$ denote terms that are further $k^{2}$ suppressed. In particular, the correction in $d=2$ is $\delta \sigma(\omega) \sim \omega \log \omega$.

Discussion and applications.-Strong renormalization of the transport parameters due to hydrodynamic fluctuations occurs if the ratios in (8) are large. When the dimensionless couplings are order unity, $\lambda_{\kappa} \sim \lambda_{D} \sim 1$ [31], the strength of fluctuations is controlled by the specific heat per "thermal volume" $c \ell_{\text {th }}^{d}$. This quantity can be thought of as the number of d.o.f. in the smallest 
volume that can reach local thermodynamic equilibrium. When there are many d.o.f. in a thermal volume, fluctuation effects are small. One might further expect that a sufficient number of d.o.f. are necessary in order for a region to locally thermalize, and hence $c \ell_{\text {th }}^{d} \gtrsim 1$. Indeed, such a bound has been established in the presence of operators with microscopic positivity properties, using the eigenstate thermalization hypothesis [32,33]. Thus, hydrodynamic fluctuation corrections to thermal transport parameters are expected to be at most comparable to the bare values.

If microscopic interactions are weak then $\ell_{\mathrm{th}} \sim \ell_{\mathrm{mfp}}$, the (inelastic) quasiparticle mean free path, will be large. Fluctuation corrections to transport are therefore small in weakly interacting systems. In contrast, in strongly correlated systems $\ell_{\text {th }}$ can become very short and fluctuations may be important. For example, at high temperatures in a lattice model, with order one d.o.f. per unit cell, the bound mentioned above is only saturated when $\ell_{\mathrm{th}} \sim a$, the lattice spacing. This roughly coincides with the "minimal" mean free path for thermal transport by well-defined phonons in insulators $[34,35]$. In strongly correlated regimes, however, the notion of a mean free path is likely not a useful concept. Recent measurements of thermal diffusivity in cuprates [36,37] and perovskites [38-40] suggest (assuming that the microscopic sound speed is the relevant velocity) that a transport length scale reaches and possibly surpasses the lattice spacing at high temperatures. The specific heat in these materials is roughly $c a^{2} \sim 40$ and $c a^{3} \sim 15$, respectively. It may be interesting to look for signatures of diffusive fluctuations in the thermal transport of these systems.

Fluctuation effects can also become important for transport close to a thermal phase transition. The thermalization length diverges as $\ell_{\mathrm{th}} \sim \tau^{-\nu}$ as the reduced temperature $\tau \rightarrow 0$, while the specific heat scales as $c \sim \tau^{2-\alpha}$. It follows that $\delta D / D \sim \tau^{\alpha+d \nu-2} \sim 1$ if hyperscaling is obeyed, so fluctuations are important in that case. Above the upper critical dimension hyperscaling is violated and fluctuations are small. A more sophisticated discussion must include fluctuations of the order parameter in the analysis [41].

Transport length scales approaching or exceeding the lattice spacing are also seen in "bad metals" [42-44]. All of our expressions above are easily adapted to describe the diffusion of a single conserved $U(1)$ charge, instead of heat [45]. Particle-hole symmetry should be broken, typically by a background charge density, otherwise many terms we have considered are forced to be zero. The correction to the dc electrical conductivity $\sigma$, e.g., is found to be

$$
\frac{\delta \sigma}{\sigma}=\frac{f_{d}}{\ell_{\mathrm{th}}^{d}} \frac{T}{\chi \mu^{2}} \lambda_{\sigma}
$$

Here, $\chi$ is the charge susceptibility and $\mu$ the chemical potential. In the definition of the couplings in (3) in terms of the thermodynamic derivatives (4), one replaces $T \rightarrow \mu$.
Condensed matter systems-including most bad metals-are typically at degenerate temperatures $T<$ $E_{F}$, below the Fermi energy. At these temperatures $\chi \sim k_{F}^{d} / E_{F}$ and $\mu \sim E_{F}$. Here, $k_{F}$ is the Fermi momentum. The contribution (16) of fluctuations to the conductivity is therefore small, even when the thermalization length becomes of order $\ell_{\text {th }} \sim a \sim 1 / k_{F}$. This is the shortest length consistent with local thermalization [33]. In contrast, at high temperatures where fermions are nondegenerate, $\chi \sim 1 /\left(T a^{d}\right)$. If the total charge is held fixed, then $\mu \sim T$. It follows that as the thermalization length becomes short, of order $\ell_{\text {th }} \sim a$, fluctuation corrections to the conductivity are order one. Diffusive transport by strongly correlated but nondegenerate fermions has recently been probed in an ultracold atom realization of the Hubbard model [46], and earlier in, e.g., [47], as well as in numerics [48,49]. Indeed, $\ell_{\text {th }}$ is found to saturate around the lattice scale at high temperatures, and so fluctuation effects may be important.

Finally, diffusion with a short thermalization length has also been seen in spin transport in strongly interacting ultracold atoms in a trap [50]. The formulas we have developed can be applied directly to longitudinal spin diffusion in a magnetic field (to break spin reversal symmetry) or to transverse spin diffusion without a magnetic field or spontaneous magnetization (so that isotropy prevents mixing of the two transverse modes). At temperatures $T \lesssim E_{F}$, with electrons on the verge of becoming nondegenerate, the thermalization length is found to be $\ell_{\mathrm{th}} \sim 1 / k_{F}$ (there is no lattice scale in these experiments). Diffusive fluctuations may therefore again be important for transport.

In summary, long wavelength fluctuations about diffusive dynamics may be relevant in condensed matter and cold atom systems of widespread interest. We have seen that a systematic derivation of these effects leads to different results than previous, more phenomenological, approaches. For this reason, it will be important to revisit the computation of fluctuations in relativistic hydrodynamics $[9,51]$, which includes a sound mode in addition to transverse momentum diffusion. Fluctuations in relativistic hydrodynamics may have direct consequences for the quark-gluon plasma.

We would like to thank Erez Berg, Debanjan Chowdhury, Paolo Glorioso, and Andrew Lucas for illuminating discussions. S. A. H. and L. V.D. are partially supported by the U.S. Department of Energy Office of Science under Award No. DE-SC0018134. X. C. L. is supported by the Knut and Alice Wallenberg Foundation.

[1] J. Fourier, Théorie Analytique de la Chaleur, par M. Fourier (Chez Firmin Didot, père et fils, Paris, France, 1822).

[2] J. Crank et al., The Mathematics of Diffusion (Oxford University Press, Oxford, 1979). 
[3] P. C. Martin, E. D. Siggia, and H. A. Rose, Phys. Rev. A 8, 423 (1973).

[4] Y. Pomeau and P. Résibois, Phys. Rep. 19, 63 (1975).

[5] M. Kardar, G. Parisi, and Y.-C. Zhang, Phys. Rev. Lett. 56, 889 (1986).

[6] I. D. Schepper, H. V. Beyeren, and M. Ernst, Physica (Utrecht) 75, 1 (1974).

[7] P. Kovtun and L. G. Yaffe, Phys. Rev. D 68, 025007 (2003).

[8] S. Mukerjee, V. Oganesyan, and D. Huse, Phys. Rev. B 73, 035113 (2006).

[9] P. Kovtun, G. D. Moore, and P. Romatschke, Phys. Rev. D 84, 025006 (2011).

[10] P. Kovtun, J. Phys. A 48, 265002 (2015).

[11] P. M. Chaikin and T. C. Lubensky, Principles of Condensed Matter Physics (Cambridge University Press, Cambridge, England, 1995), Vol. 1.

[12] S. Dubovsky, L. Hui, A. Nicolis, and D. T. Son, Phys. Rev. D 85, 085029 (2012).

[13] S. Grozdanov and J. Polonyi, Phys. Rev. D 91, 105031 (2015).

[14] F. M. Haehl, R. Loganayagam, and M. Rangamani, J. High Energy Phys. 05 (2015) 060.

[15] M. Crossley, P. Glorioso, and H. Liu, J. High Energy Phys. 09 (2017) 095.

[16] K. Jensen, N. Pinzani-Fokeeva, and A. Yarom, J. High Energy Phys. 09 (2018) 127.

[17] H. Liu and P. Glorioso, Proc. Sci. TASI2017 (2018) 008.

[18] X. Han and S. A. Hartnoll, Phys. Rev. Lett. 121, 170601 (2018).

[19] A. Lucas, arXiv:1809.07769.

[20] P. Gao, P. Glorioso, and H. Liu, arXiv:1803.10778.

[21] See Supplemental Material at http://link.aps.org/ supplemental/10.1103/PhysRevLett.122.091602, which includes Refs. [22-27], for a review of the formalism of Ref. [15] and its application to loop corrections to diffusion.

[22] S. Endlich, A. Nicolis, R. A. Porto, and J. Wang, Phys. Rev. D 88, 105001 (2013).

[23] M. Blake, H. Lee, and H. Liu, J. High Energy Phys. 10 (2018) 127.

[24] L. V. Delacrétaz and S. A. Hartnoll, Phys. Rev. B 97, 220506 (2018).

[25] T. Banks and A. Lucas, Phys. Rev. E 99, 022105 (2019).

[26] K. Jensen, R. Marjieh, N. Pinzani-Fokeeva, and A. Yarom, SciPost Phys. 5, 053 (2018).

[27] E. Wang and U. W. Heinz, Phys. Rev. D 66, 025008 (2002).

[28] That these interactions should arise is already clear from the stochatistic approach, where the fluctuation dissipation theorem imposes $\langle\xi(x) \xi\rangle=-2 T^{2} \kappa(\varepsilon) \nabla^{2} \delta(x)$. These interactions only vanish if $\kappa(\varepsilon)=$ const, which is also apparent in (S.21).

[29] P. Kovtun, J. Phys. A 45, 473001 (2012).
[30] Additional poles in (6) are outside the validity of the resummation. The nonanalytic corrections to diffusion are seen to be more important than the $O\left(k^{4}\right)$ higher derivative corrections to the diffusion equation in $d=1$ and also in $d=2$, where the dispersion receives an additional imaginary part $O\left(k^{4} \log k\right)$.

[31] Using Eqs. (12) and (S.21), this holds in any scaling regime where $\kappa \sim T^{a}$ and $c \sim T^{b}$.

[32] L. V. Delacrétaz, T. Hartman, S. A. Hartnoll, and A. Lewkowycz, J. High Energy Phys. 10 (2018) 028.

[33] X. Chen-Lin, L. V. Delacrétaz, and S. A. Hartnoll (to be published).

[34] G. A. Slack, Solid State Phys. 34, 1 (1979).

[35] P. B. Allen, X. Du, L. Mihaly, and L. Forro, Phys. Rev. B 49, 9073 (1994).

[36] J. C. Zhang, E. M. Levenson-Falk, B. J. Ramshaw, D. A. Bonn, R. Liang, W. N. Hardy, S. A. Hartnoll, and A. Kapitulnik, Proc. Natl. Acad. Sci. U.S.A. 114, 5378 (2017).

[37] J. Zhang, E. D. Kountz, E. M. Levenson-Falk, R. L. Greene, and A. Kapitulnik, arXiv:1808.07564.

[38] A. M. Hofmeister, J. Appl. Phys. 107, 103532 (2010).

[39] V. Martelli, J. L. Jiménez, M. Continentino, E. BaggioSaitovitch, and K. Behnia, Phys. Rev. Lett. 120, 125901 (2018).

[40] K. Behnia, A. Kapitulnik et al. (to be published).

[41] P. C. Hohenberg and B. I. Halperin, Rev. Mod. Phys. 49, 435 (1977).

[42] V. J. Emery and S. A. Kivelson, Phys. Rev. Lett. 74, 3253 (1995).

[43] O. Gunnarsson, M. Calandra, and J. E. Han, Rev. Mod. Phys. 75, 1085 (2003).

[44] N. E. Hussey, K. Takenaka, and H. Takagi, Philos. Mag. 84, 2847 (2004).

[45] If thermoelectric effects are strong, one should instead work with coupled heat and charge diffusion. This is done in the Supplemental Material [21].

[46] P. T. Brown, D. Mitra, E. Guardado-Sanchez, R. Nourafkan, A. Reymbaut, S. Bergeron, A.-M. S. Tremblay, J. Kokalj, D. A. Huse, P. Schauss, and W. S. Bakr, Science 363, 379 (2019).

[47] U. Schneider, L. Hackermüller, J. P. Ronzheimer, S. Will, S. Braun, T. Best, I. Bloch, E. Demler, S. Mandt, D. Rasch, and A. Rosch, Nat. Phys. 8, 213 (2012).

[48] C. H. Mousatov, I. Esterlis, and S. A. Hartnoll, arXiv: 1803.08054.

[49] E. W. Huang, R. Sheppard, B. Moritz, and T. P. Devereaux, arXiv:1806.08346.

[50] T. Enss and J. H. Thywissen, arXiv:1805.05354.

[51] M. Stephanov and Y. Yin, Phys. Rev. D 98, 036006 (2018). 